\title{
Effect of ground electrode on charge injection and surface potential of corona charged polyethylene film
}

\author{
Yuan Zhuang ${ }^{1}$, Miao Hao ${ }^{*}$, George Chen ${ }^{1}$, Xin Chen ${ }^{4}$, Chong Zhang ${ }^{2,4}$, Wenpeng Li ${ }^{3,4}$, \\ Haitian Wang ${ }^{4}$, Mingyu Zhou ${ }^{4}$, Xianzhang Lei ${ }^{4}$ \\ ${ }^{1}$ University of Southampton, University Road, Southampton, SO17 2GR, United Kingdom \\ ${ }^{2}$ School of Chemistry and Biological Engineering, University of Science \& Technology Beijing, Beijing \\ 100083, P. R. China \\ ${ }^{3}$ State Key Laboratory of Electrical Insulation and Power Equipment, Xi'an Jiaotong University, Xi' an \\ 710049, China \\ ${ }^{4}$ Global Energy Interconnection Research Institute, Beijing, 102200, China
}

${ }^{*}$ Corresponding author: e-mail address: m.hao@soton.ac.uk

\begin{abstract}
The surface potential decay measurement is a simple and low cost tool to assess electrical properties of insulation materials; therefore, understanding the physical mechanisms of the surface potential decay becomes necessary. With our recent space charge measurement results on corona charged samples, bipolar charge injection on corona charged samples had been observed. Based on this new fact, it is anticipated that the ground electrode should have significant effect during corona charging and subsequently decay processes. In the paper, low density polyethylene (LDPE) film with gold ground electrode was compared with LDPE film with aluminium ground to study effect of ground electrode on charge injection and surface potential decay processes. Charging current during the corona charging, surface potential decay and space charge dynamics after corona charging in the samples with either gold coated or aluminium ground electrode were measured. Differences have been observed for gold ground electrode when compared with aluminium ground electrode. Higher work function of gold electrode is responsible for the observed differences. A preliminary simulation has also attempted to show that the bipolar injection may take place in corona charged LDPE films.
\end{abstract}

\section{Introduction}

The study of surface potential decay in dielectric materials has a long history and is closely related to the wide application of corona charged dielectrics. It became a very popular topic after the surface potential decay cross-over phenomenon was found in 1967 [1]. To understand the physical mechanisms that may cause it, several assumptions and hypothesis have been made and the explanation so far is not satisfactory. Generally, there are four possible mechanisms that have been discussed in relation to their effects over the surface potential decay process: gas neutralization, surface conduction, sample polarization and charge injection [2]. Most hypotheses believe that the effect of gas neutralization and surface conduction can only be applied in some special circumstances and neglected in conventional condition; the sample polarization is also negligible if the charging period is long enough [3-7]. The deep trap on the surface and shallow trap in the material was used to qualitatively explain the cross-over phenomenon; when corona discharge charged the polymer, the surface of the film is at a high potential and electrons travel easily across the deep trap due to higher energy, therefore, electric charge on the surface decays faster. If the film is less charged by corona discharge, and the surface is at a low potential, 
then the electron with less total energy cannot travel across the deep trap easily [8]. To understand clearly how the corona charged film decays, not only the charge injection at the surface need to be studied, but also knowledge on the charge distribution inside the polymer film is required.

Over the last 20 years, there has been significant development in space charge mapping in solid dielectrics due to the advances in sensors, signal capture and processing. Techniques such as the laserinduced pressure pulse method (LIPP) [9], thermal step method (TS) [10] and pulsed electroacoustic method (PEA) [11] have been used to study in the field of space charge measurement. They provided significant assistance in the understanding of charge injection, charge transport and trapping (detrapping). Previous work [12] applied the PEA technique to corona charged sample and therefore observed the bipolar charge injection and the decay process inside the polymer film. The aim of this paper is to extend our understanding of occurrence of bipolar charge injection by investigating the influence of different ground electrodes, i.e. aluminium and gold.

\section{Experimental Details}

\subsection{Sample preparation}

The samples used for the decay experiments are low density polyethylene (LDPE) film with $50 \mu \mathrm{m}$ thickness. The film was purchased commercially from GoodFellow. Additive-free LDPE was selected to avoid extra complications that might be caused by the presence of antioxidants and other additives. The film was cut into a circular disc with a diameter of $55 \mathrm{~mm}$, cleaned initially using methanol, rinsed in deionized water and then dried in air. For gold coated sample, the gold electrode was sputtered on one side of the sample by K500X Sputter coater for 2 min and 30 seconds using a $25 \mathrm{nA}$ current. The settings give $20 \mathrm{~nm}$ thick with $53 \mathrm{~mm}$ diameter gold on one side of the polymer.

\subsection{Surface potential decay measurement}

The LDPE film was negatively charged in the needle-grid corona charging system shown in Figure 1. The needle electrode is $3 \mathrm{~cm}$ away from the top surface of earth plate. It always has a relatively high voltage to generate corona discharge. The grid electrode is $1.5 \mathrm{~cm}$ away from the top surface of earth plate. It was used to control the surface potential of the tested samples and uniformly distributed the potential along the whole surface of the sample. The grid potential is influenced by the needle potential. If the grid potential is selected too low, then its value will be controlled by the needle potential instead of its voltage source. All the results shown in this paper are referenced by the grid electrode voltage. As the earth plate is made from aluminium, the bottom surface of the sample will be in direct contact to it. Therefore, it is known as the aluminium electrode sample. There are two ways to build gold electrode samples; one is that the gold coated on one side of the LDPE sample and the sample will contact the aluminium earth plate, the other is that the gold coated on the aluminium earth plate. After the charging voltage has been set up, the sample described in section 2.1 will be charged under the grid electrode for a fixed 2 min charging time. To achieve consistent results, all experiments were carried out in a controlled environment where temperature and relative humidity were kept at $23{ }^{\circ} \mathrm{C}$ and $20 \%$ respectively.

Once charged, the sample was transferred to a compact JCI 140 static monitor to observe the surface potential decay. The time required to transfer the sample is around $1 \mathrm{~s}$, and the first decay data is taken at $5 \mathrm{~s}$ after the charging due to the time interval of the static monitor taken a reading. The user manual of JCI 140 static monitor shows that the reading from the instrument varies with the distance between its sensor and the measured object. Therefore, to convert the readings from the static monitor into the sample surface potentials, a calibration needs to be carried out. The static monitor in our experiment is set $3 \mathrm{~cm}$ above the sample top surface. The calibration was done by placed a $5 \mu \mathrm{m}$ thick with $53 \mathrm{~mm}$ diameter aluminium foil which connect with a high voltage source on top of our sample. A linear 
relationship with $R^{2}=0.998$ is obtained between the reading from the static monitor and the applied voltage to the aluminium foil as shown in Figure 2. Therefore, according to the equation shown in Figure 2 , the surface potential on the LDPE film can be evaluated by using the readings from the static monitor divided by a factor of 1.692. Of course, the slope in the linear relationship will change with the geometry of the experimental settings. However the thickness of the gold is negligible, hence the surface potential from the films with gold electrode can be measured under the same calibration.

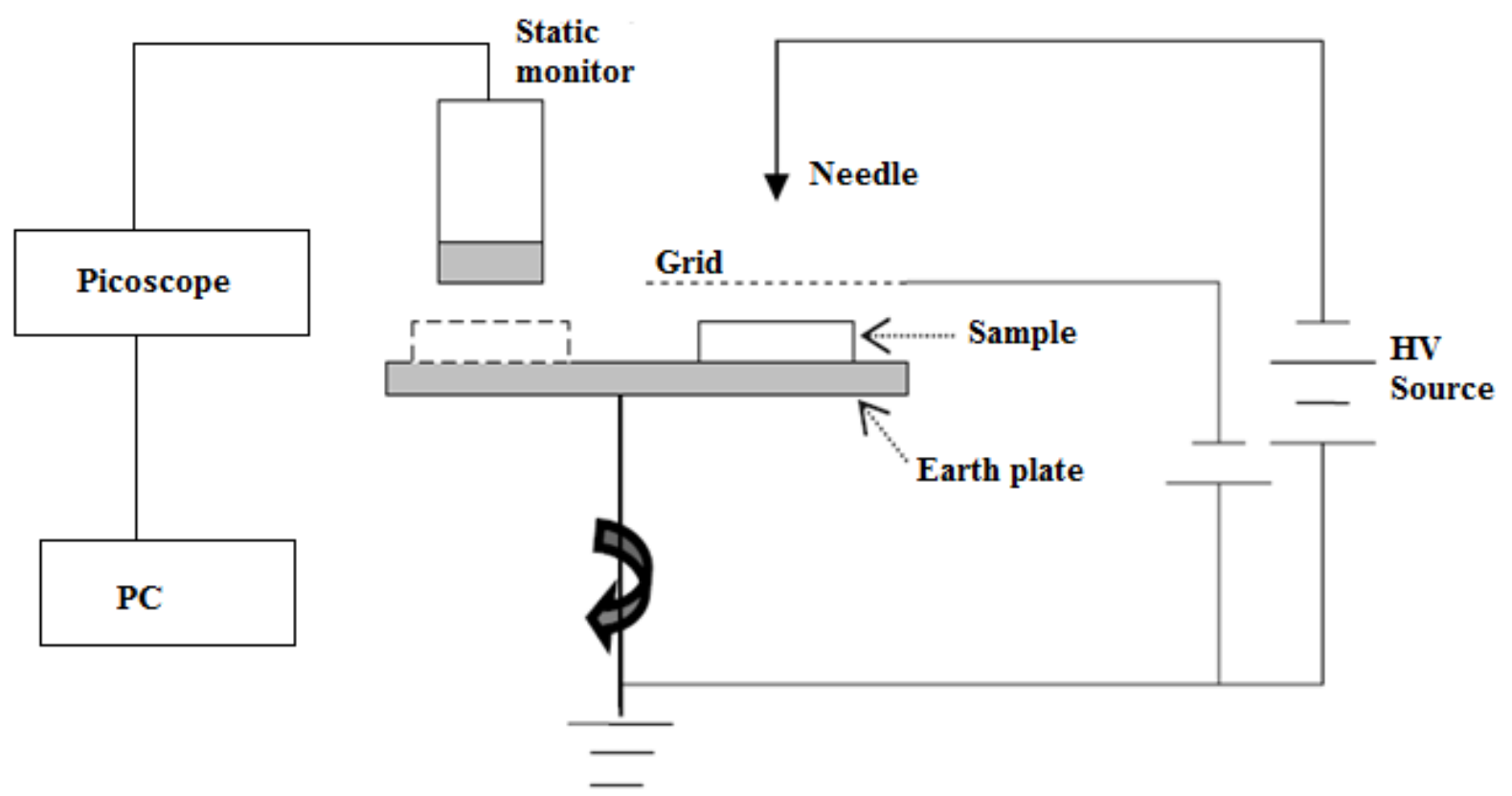

Figure 1. Experiment set up for corona charging and potential decay measurement system

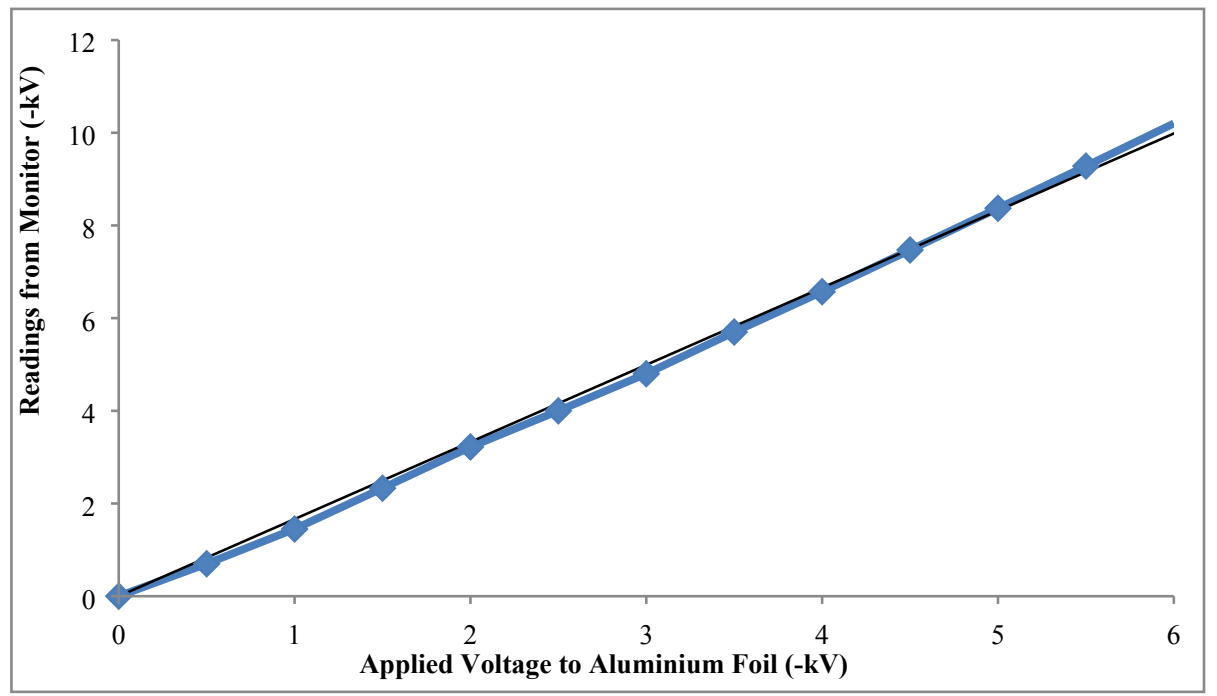

Figure 2. Calibration of JCI 140 static monitor

\subsection{Corona charging current measurement}

Considering the large size of the grid electrode $(9 \mathrm{~cm} \times 4.5 \mathrm{~cm})$, the corona charging current was measured by using a $50 \mu \mathrm{m}$ thick with $10 \mathrm{~cm}$ diameter LDPE. The charging current was determined by measuring the voltage across a $10 \mathrm{M} \Omega$ resistor placed underneath the earth plate. As the sample is big, gold is coated on the aluminium earth plate to provide the gold electrode for the current measurement. It is known that the distance between the grid electrode and the sample will affect the sample's potential. 
In the present study, the grid electrode is set to $1.5 \mathrm{~cm}$ away from the ground therefore the thickness of the gold is negligible. The currents were measured during corona charging period.

\subsection{Space charge measurement}

The space charge distribution in the corona charged LDPE film was measured by the pulsed electroacoustic (PEA) technique. This technique is widely used due to its simple structure, low cost and ease of implementation. After one LDPE film was corona charged for 2 min in the corona charging system, it was carefully removed by using a pair of insulated head tweezers. To protect the charges on both side of the sample, it needs to be sandwiched by two additional LDPE films $A$ and $B$. which are made from the same material as described in section 2.1. The typical experimental procedure is described as follows: (i) place film B on the ground electrode in the PEA system, (ii) transfer the corona charged film and put it on top of film B, and finally (iii) place film A on top of the corona charged sample before mounting the PEA head. The whole process takes about 1 minute before the first PEA measurement can be carried out. Figure 3 shows the setup of PEA measurement and Figure 4 shows that the gold coated sample is still gold coated while doing the PEA measurement. A very thin layer of silicone oil is often smeared on the electrodes to improve acoustic coupling. When a voltage pulse of $5 \mathrm{~ns}$ length is applied to the sample sandwiched between the two electrodes, the pulse electric field interacts with the charges on the electrodes as well as the accumulated space charges in the specimen, and the movement of charge generates acoustic waves. The acoustic wave produced corresponds to each charged layer. The waves are then transmitted to the bottom electrode, which is much thicker hence it will delay the arrival of the waves until the instability from the pulse source disappears, and then they are detected by a piezoelectric transducer. The waves are converted into an electrical signal by the transducer. The electrical signal is then amplified and finally captured with a digital oscilloscope.

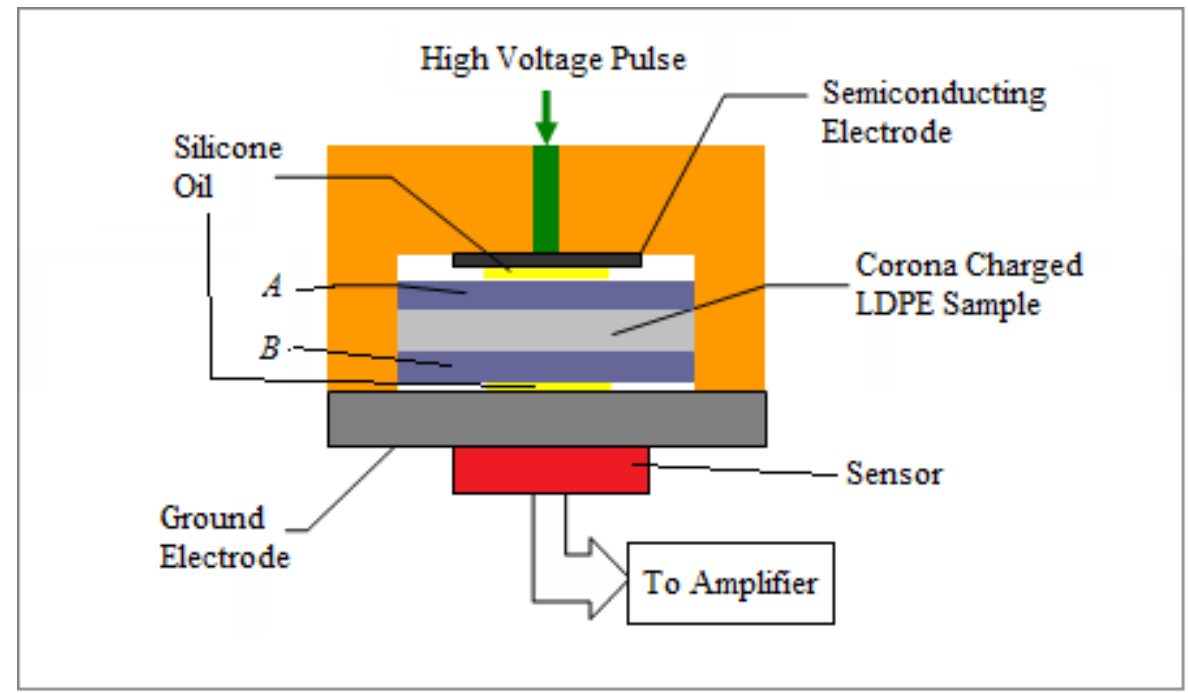

Figure 3. Sample arrangement details in PEA setup

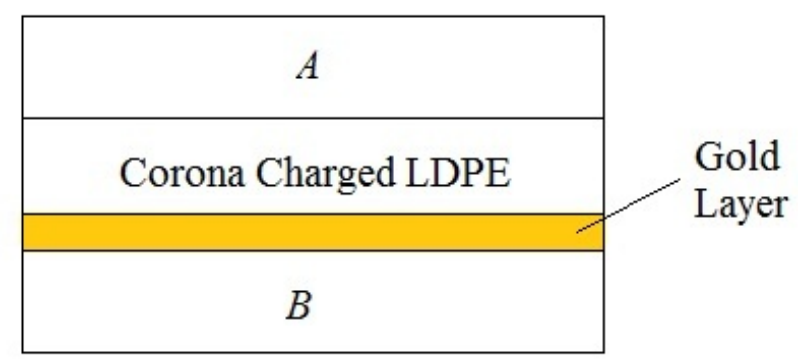


Figure 4. Space charge measurement set up for gold ground sample

\section{Experimental Results and Discussion}

\subsection{Surface potential decay measurement}

In this section, the surface potential decay results measured by the static monitor will be discussed. Negative polarity was used to deposit charges on the sample surface; however, the absolute surface potential has been used here. It has been observed that the surface potential decay curves are almost the same for gold coated sample and sample placed on gold coated ground electrode, only the results obtained from the gold coated sample are shown here for comparison with aluminium ground sample. From Figure 5, the difference between the gold ground and aluminium ground LDPE films can be clearly seen especially with high initial surface potential. The cross-over phenomenon is shown with the aluminium LDPE in a short decay period (at about $4 \mathrm{~min}$ ). However, for gold ground LDPE it shifts to a much longer time and it cannot be observed after 20 min decay. To analyse the effect of different ground electrode, a decay rate $D$ is introduced. $D$ is defined as:

$$
D=\frac{V\left(t_{0}\right)-V(t)}{V\left(t_{0}\right)} \times 100 \%
$$

where $V\left(t_{0}\right)$ and $V(t)$ are the potentials for the initial potential and the potential after certain time respectively. The detailed decay rates at $20 \mathrm{~min}$ for both samples and selected voltage levels are plotted in Figure 6. The decay rate D increases with the initial surface potential but not in a linear formation, indicating a complex mechanism involved. It is clearly shown that the gold ground samples have a slower decay compared with aluminium ground samples under all the voltage levels.

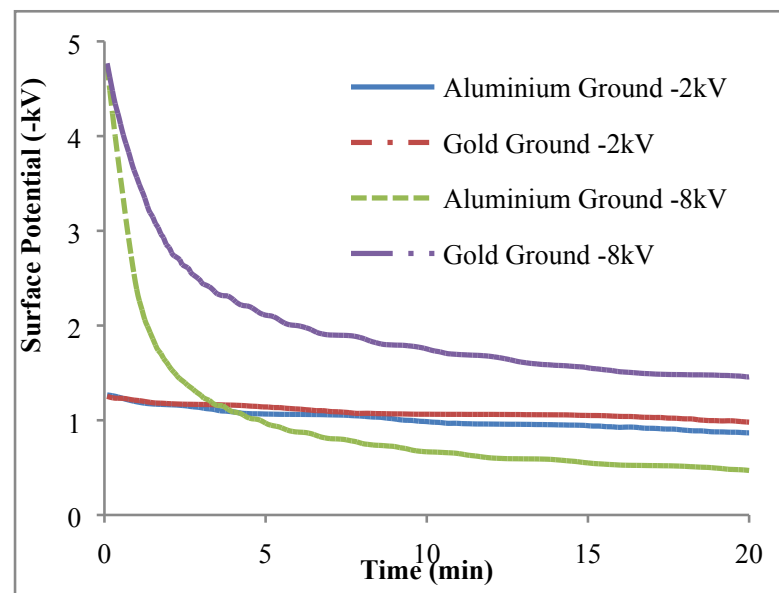

Figure 5. Surface potential decay for aluminium ground sample and gold ground sample

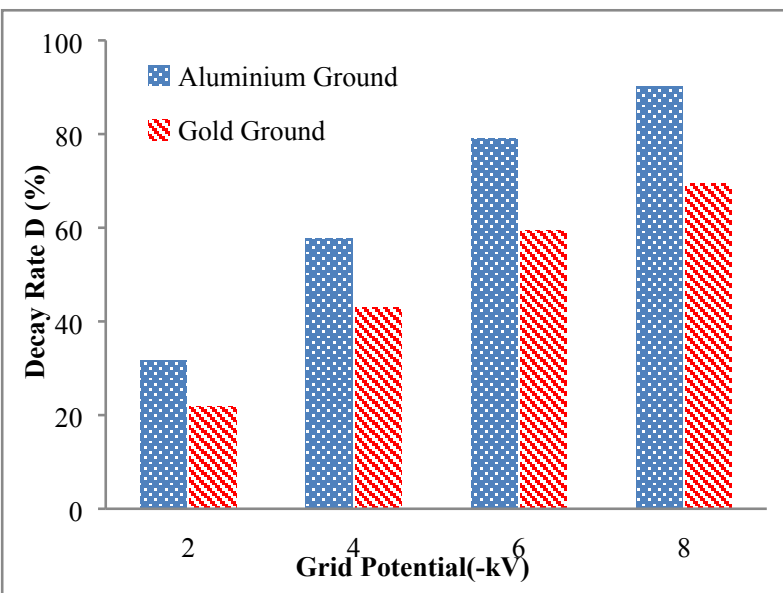

Figure 6. Surface potential decay rate for aluminium ground sample and gold ground sample at $20 \mathrm{~min}$

\subsection{Corona charging current measurement}

Figure 7 shows the corona charging current that was calculated from the measured voltage across the 10 $\mathrm{M} \Omega$ resistor. The current below $-3 \mathrm{kV}$ is too noisy to be measured in the present arrangement. The initial peak current is not shown in Figure 7 mainly because of very long charging time, however, our interest lies in the steady state of this current. To quantify the difference, the ratio of gold ground current (GGC) and aluminium ground current (AGC) at $120 \mathrm{~s}$ charging time is shown in Figure 8.

$$
\text { Ratio }=\frac{G G C}{A G C} \times 100 \%
$$


It can be seen that the measured current in the gold ground electrode LDPE films is smaller than the current of aluminium ground LDPE films at any voltage levels, and surprisingly, the ratio between these currents are around $75 \%$ for all the voltage levels measured. This can be explained by the differences of the charge injection barriers between aluminium and gold electrodes in the Schottky injection mechanism.

\subsection{Space charge measurement}

It is clear from the above results that the charge injection from the bottom layer affects the surface potential decay and the corona charging current. To reveal the mechanism that may be responsible for the observed phenomenon, charge distribution in corona charged LDPE with different electrodes were measured as shown in Figures 9 and 10 at $-2 \mathrm{kV}$ and $-8 \mathrm{kV}$ respectively. Based on the setup in Figure 3 , there are two distinctive charge troughs and peaks across the aluminium ground sample from left to right and one trough and two peaks for gold ground sample. For the aluminium ground sample, the first trough and last peak are known as the induced charge parks on the PEA electrodes, the first peak corresponds to the bottom surface of the corona charged film and the second trough represents the charges from the corona charged side of the sample. For gold ground sample, the first induced charge trough is not shown because the gold coated side of the sample is grounded during corona charging process. Therefore, based on the measuring set up in Figure 4, there is no electric field in LDPE film B, and the first trough disappeared.

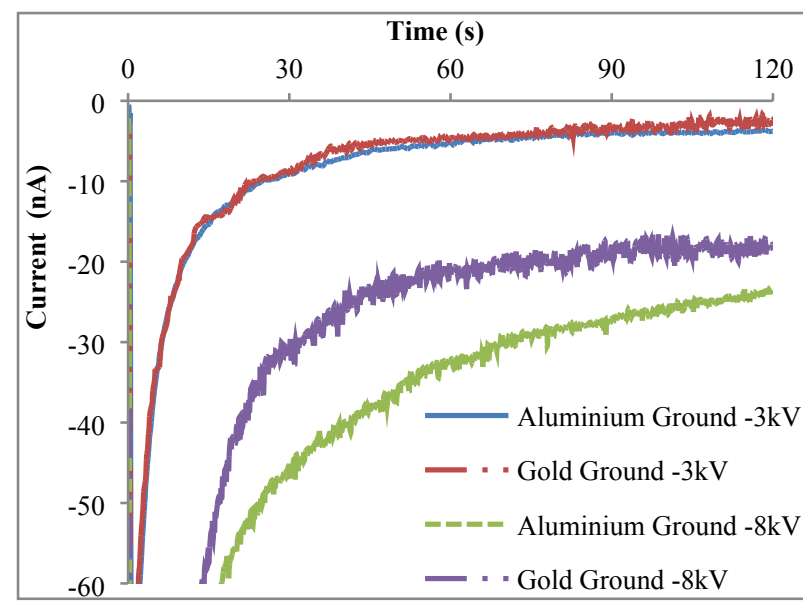

Figure 7. Corona charging current for aluminium ground sample and gold ground sample

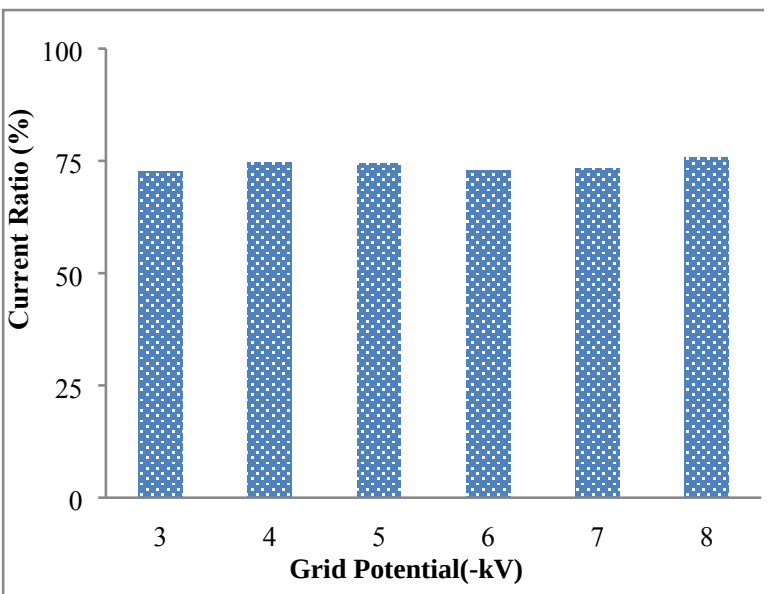

Figure 8. Corona charging current ratio versus grid potential for aluminium ground sample and gold ground sample at $2 \mathrm{~min}$ 


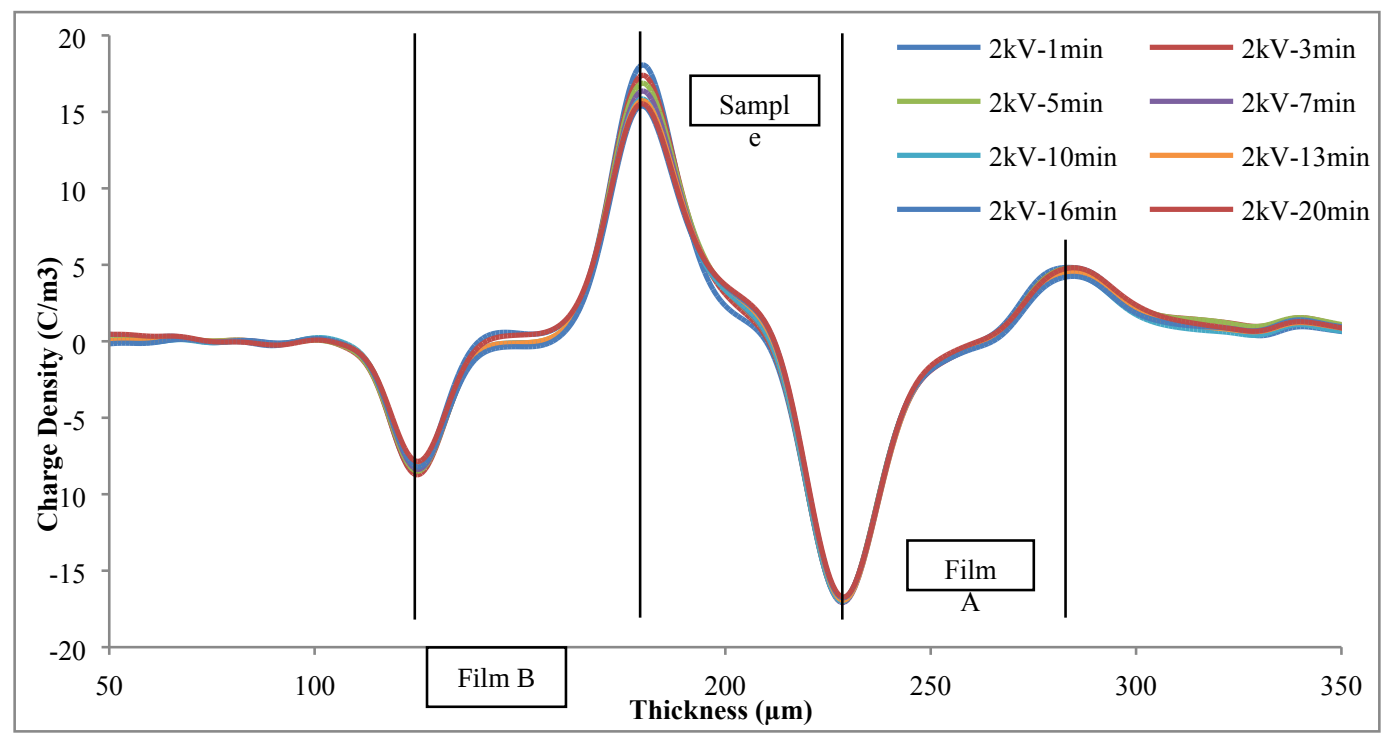

Figure 9 a). Space charge distribution for aluminium ground sample at $-2 \mathrm{kV}$

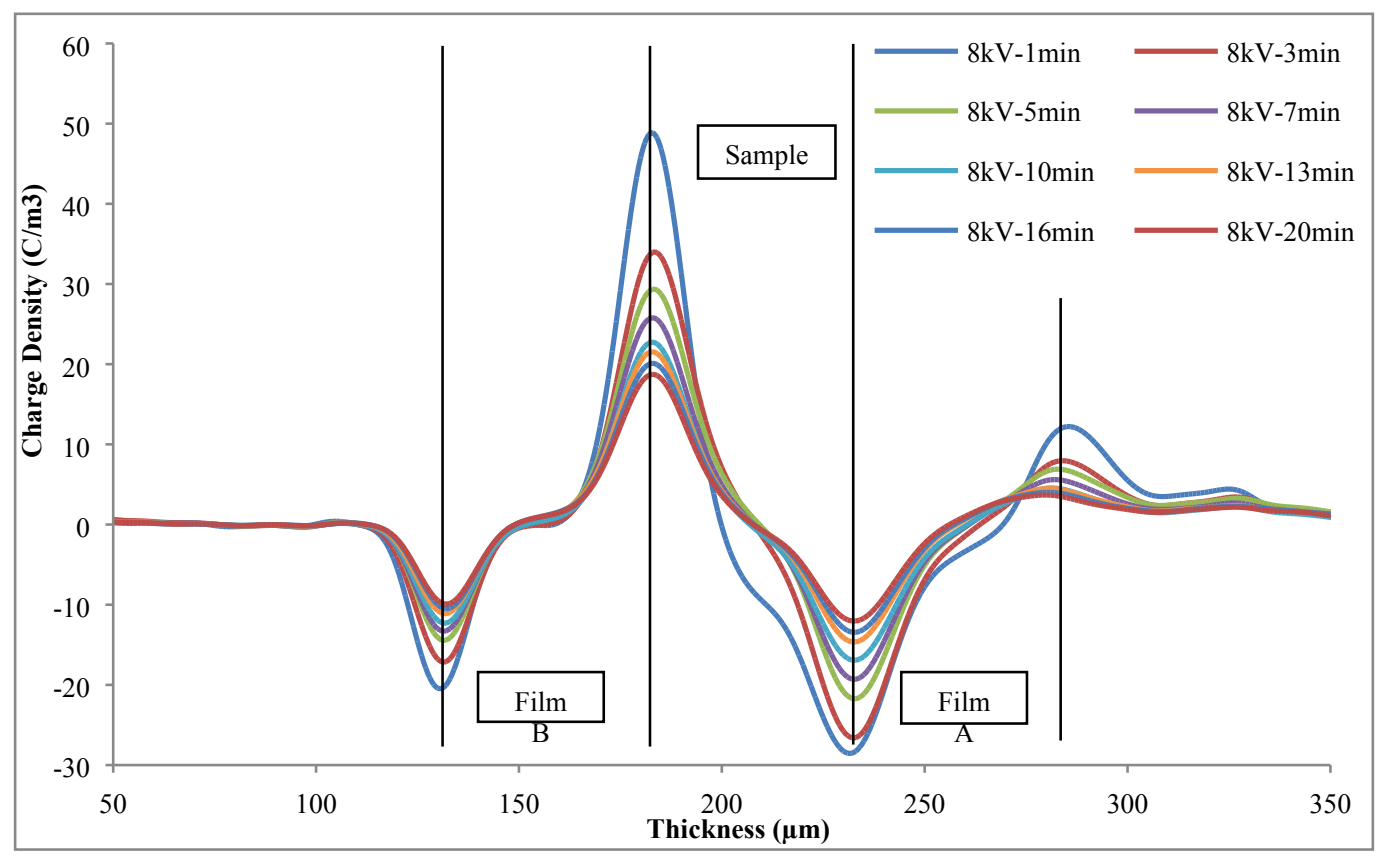

Figure 9 b). Space charge distribution for aluminium ground sample at $-8 \mathrm{kV}$ 


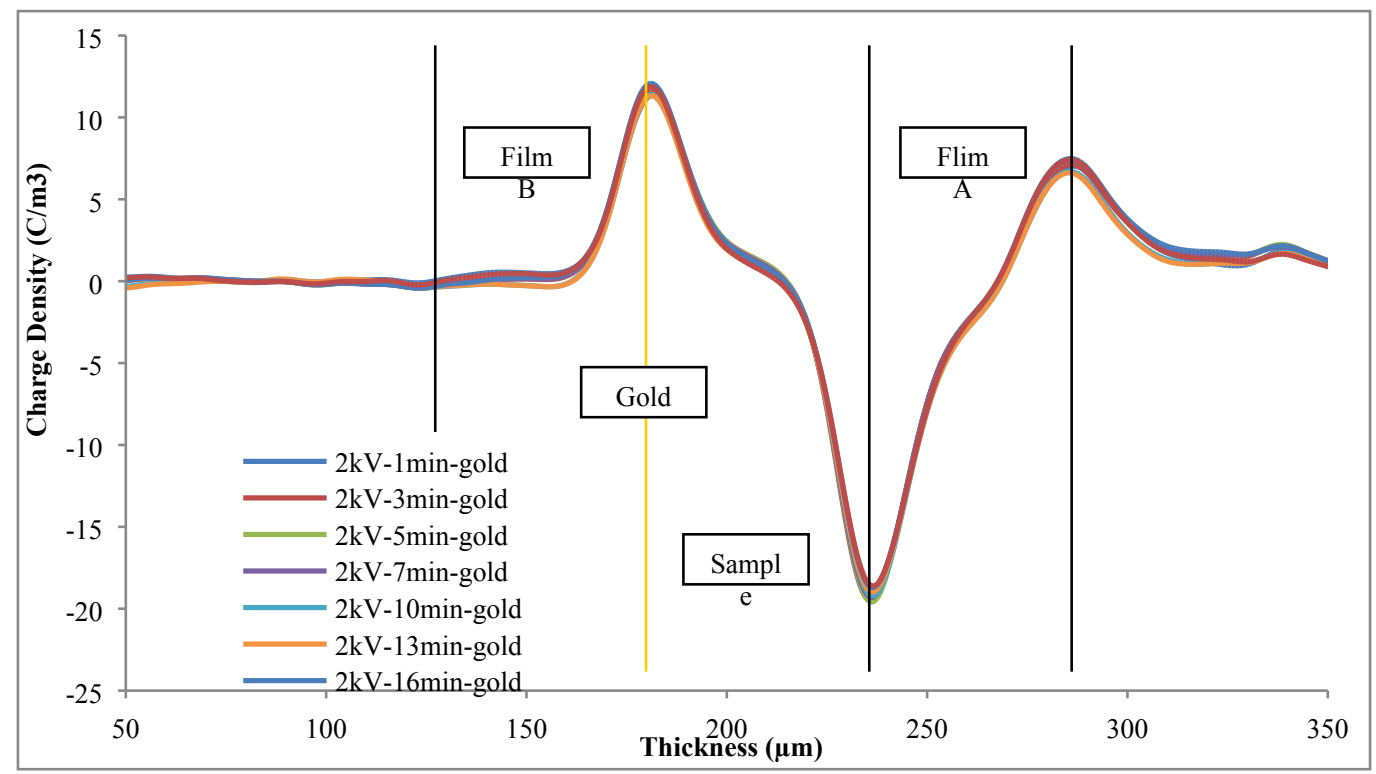

Figure 10 a). Space charge distribution for gold ground sample at $-2 \mathrm{kV}$

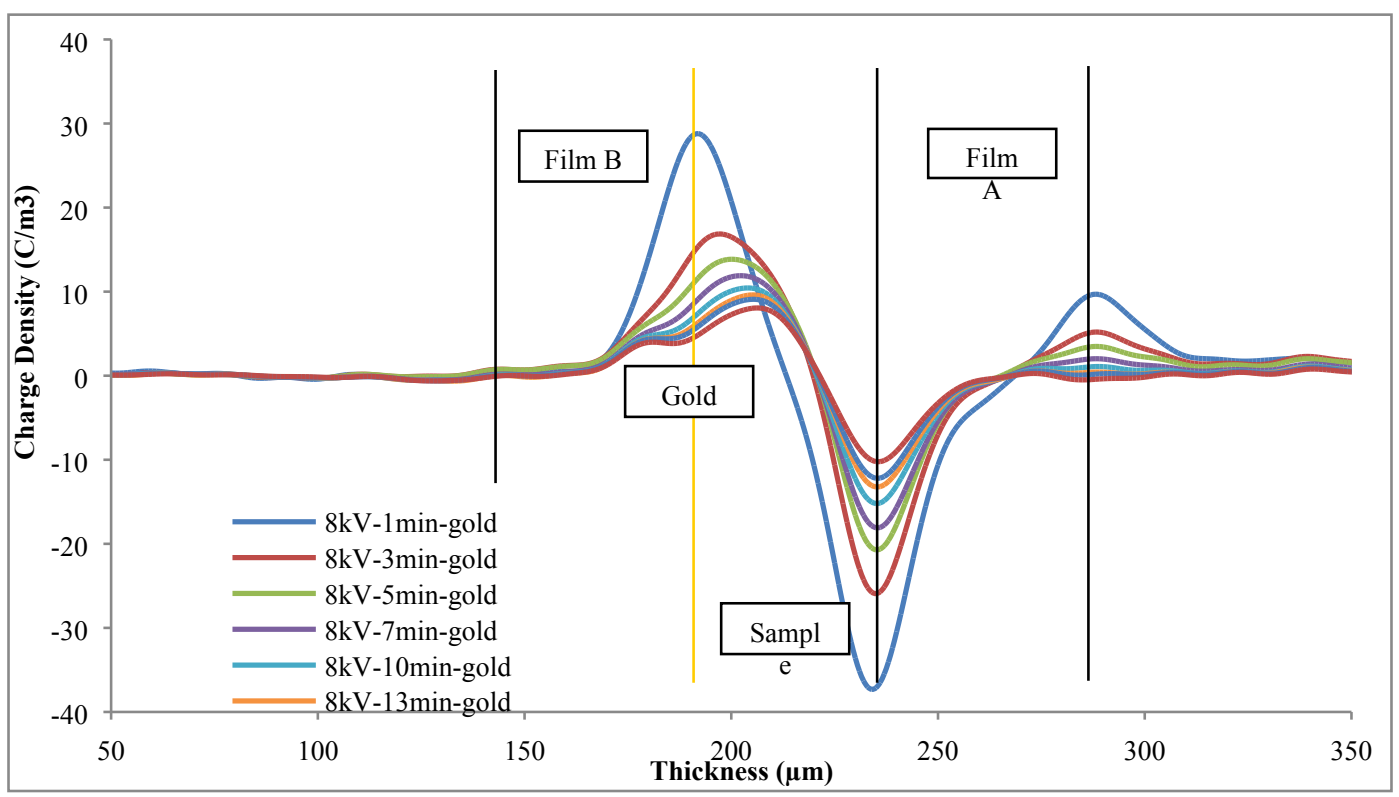

Figure 10 b). Space charge distribution for gold ground sample at $-8 \mathrm{kV}$

\subsection{Discussion}

Polyethylene is a typical semi-crystalline material; it contains various charge traps [13]. Therefore, it is believed that the injected charge can be captured by traps on its way towards to the opposite electrode. Charge injection has been observed to occur in LDPE above a threshold value of $10 \mathrm{kV} \mathrm{mm}^{-1}$ [14]. From the surface potential in Figure 5, the lowest electric field is $25 \mathrm{kV} \mathrm{mm}^{-1}$, which is well above the threshold value and therefore charge injection dominates all the voltage level in this study. By selecting different ground electrode of the sample, the differences in the decay rate in section 3.1, the corona charging current in section 3.2 and the charge density on the bottom surface of the corona charged sample in section 3.3 can be clearly observed. The work function for gold (Au) is $5.1-5.47 \mathrm{eV}$ and for aluminium (Al) is $4.06-4.26 \mathrm{eV}$ [15] i.e. gold has a higher work function than aluminium. This means that charge exchange between aluminium and polymer is easier than gold. Therefore, it explains why 
different bottom ground electrode can produce differences in the results and the injection from the bottom surface of the sample during corona charging must be taken into account.

There are few common features that can be found by comparing Figures 9 and Figure 10. The first one is that at any voltage levels, electrons and holes are injected into the sample deeply and recombined with each other. As a result, the amount of charge injection can be observed from the corona charged sample's surfaces. As the grid voltage increases, the charge densities on both the bottom surface and the top surface of the corona charged sample increase, which indicates the field dependent mechanism of injection for both surfaces. The second common feature is that the bottom surface always has more charge injection than the top surface for the aluminium ground sample; and it has less injected charge in the gold ground sample. The reason that all the gold ground samples have a larger top surface injection is because all the reading are taken $1 \mathrm{~min}$ decay, the slower decay for gold ground samples caused more charges on the top surface. The surprising observation is that the decay rate on the top surface of corona charged sample is almost the same for either the aluminium ground sample or the gold ground sample; however, there is a big difference on the decay rate of the bottom surface. This indicates that the injection on the corona charged surface is not affected by changing the ground type of the LDPE film. However, the injection from the bottom surface is reduced; the decaying rate of the charges on bottom surface is much slower and therefore reduces the top surface potential decay rate. Therefore, a preliminary simulation is attempt to prove this observed phenomenon.

\section{Simulation}

Recently, the bipolar charge transport model has been widely used to describe the current-voltage characteristics and charge dynamics in polymeric insulation after proposed in 1994 [16]. The model contains three important components: charge build-up (or generation), charge transport process with trapping/de-trapping and charge recombination under DC voltage. It has also been reported that bipolar charge transport model can be used to simulate surface potential decay on corona charged polymeric materials $[17,18]$. The numerical model can explain the influence of the bottom electrode on injection extraction at the interface during corona charging process and subsequently affect its surface potential decay results. Details of the model can be found in Reference [17].

The selection of parameters used in the model can be found in Table 1. To prove that the gold ground can play an important role in both of the charging and decaying process, all the parameters are kept the same expect the bottom surface injection barrier height from the Schottky injection, which is higher for gold electrode due to its higher work function compared with aluminium [15].

The preliminary simulation results for aluminium ground sample and gold ground sample are shown in Figure 11. It can be clearly seen that the surface potential with gold ground sample at both $-2 \mathrm{kV}$ and $-8 \mathrm{kV}$ decay slower than that with aluminium ground sample. Also the cross-over phenomenon can be observed for aluminium ground sample and no cross-over is shown for the gold ground sample. These results prove that reducing the amount of charge injection from the bottom layer of the corona charged sample can reduce its corona charged surface potential decay rate.

Table 1. Simulation Parameters

\begin{tabular}{|c|c|}
\hline Parameters (Unit) & Value \\
\hline$w_{e i}(\mathrm{eV})$ & 1.1 \\
\hline
\end{tabular}




\begin{tabular}{|c|c|}
\hline Aluminium $w_{h i}(\mathrm{eV})$ & 1.1 \\
\hline Gold $w_{h i}(\mathrm{eV})$ & 1.2 \\
\hline Electrons/Holes Mobility $\left(\mathrm{m}^{2} \mathrm{~V}^{-1} \mathrm{~s}^{-1}\right)$ & $9 \times 10^{-15}$ \\
\hline Electrons trapping coefficient $\left(\mathrm{s}^{-1}\right)$ & $7 \times 10^{-3}$ \\
\hline Holes trapping coefficient $\left(\mathrm{s}^{-1}\right)$ & $7 \times 10^{-3}$ \\
\hline $\mathrm{S}_{0}\left(\mathrm{~m}^{3} \mathrm{C}^{-1} \mathrm{~s}^{-1}\right)$ & $4 \times 10^{-3}$ \\
\hline $\mathrm{S}_{1}\left(\mathrm{~m}^{3} \mathrm{C}^{-1} \mathrm{~s}^{-1}\right)$ & $4 \times 10^{-3}$ \\
\hline $\mathrm{S}_{2}\left(\mathrm{~m}^{3} \mathrm{C}^{-1} \mathrm{~s}^{-1}\right)$ & $4 \times 10^{-3}$ \\
\hline $\mathrm{S}_{3}\left(\mathrm{~m}^{3} \mathrm{C}^{-1} \mathrm{~s}^{-1}\right)$ & 0 \\
\hline Relative permittivity of LDPE & 2.3 \\
\hline
\end{tabular}

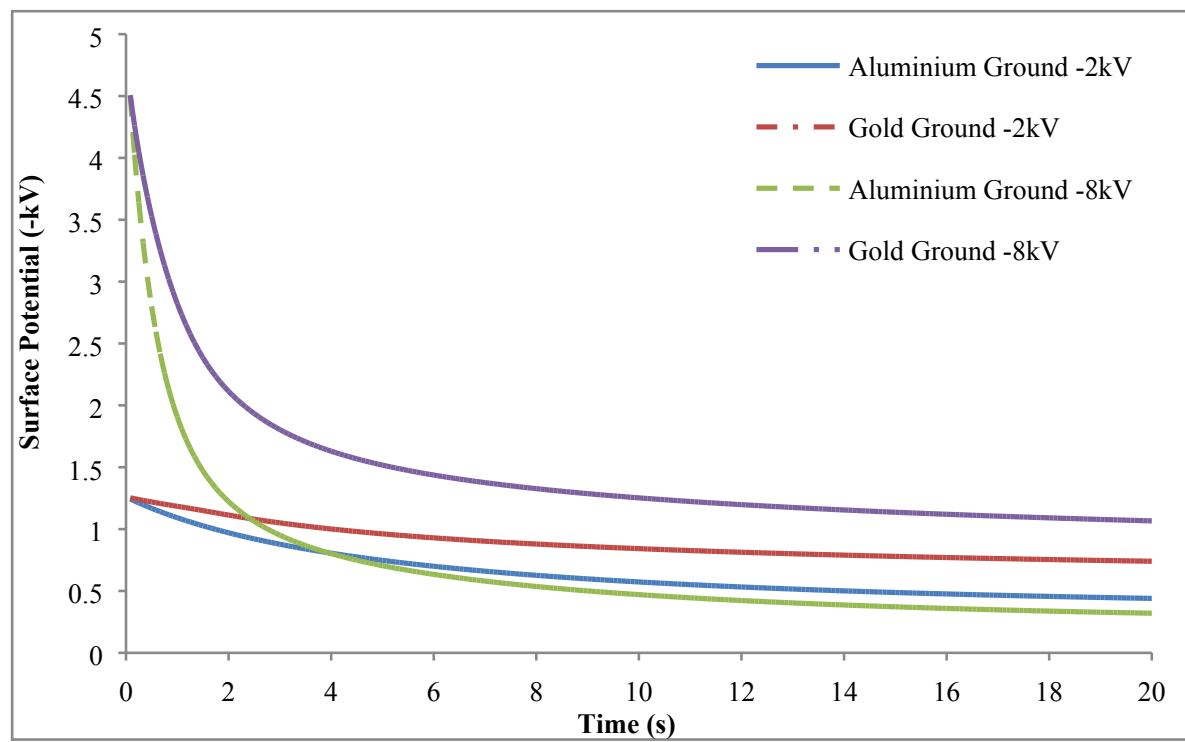

Figure 11. Preliminary simulation results for aluminium ground sample and gold ground sample

\section{Conclusions}

The effect of ground electrode on charge injection and surface potential decay of corona charged LDPE has been studied using four different techniques: surface potential decay measurement, corona charging current measurement, space charge measurement and bipolar charge transport model. The surface potential decay measurement shows clearly the different decay rates of the aluminium ground sample and the gold ground sample. The corona charging current measurement shows that the ground electrode injection from the gold electrode is always smaller than the aluminium ground electrode injection. The space charge measurement clarifies the charge density along both top surface and bottom surface of the sample. Finally, the simulation results prove that the reduction of the bottom surface injection can make the top surface potential decaying slower. Combining all the results, it can be concluded that bipolar charge injection is the key factor during the corona charging and decaying process and the injection from the bottom surface of the sample must be taken into account. 


\section{Acknowledgements}

The authors are grateful for the financial support from the State Grid Cooperation of China: Science and Technology Project of SGCC(SGRIZLJS[2014]888).

\section{References}

[1] Ieda M, Sawa G and Shinohara I 1967 A decay process of surface electric charges across polyethylene film J. Appl. Phys. 6 793-4

[2] Molinié P 1999 Charge injection in corona-charged polymeric films: potential decay and current measurements Journal of Electrostatics 45 265-273

[3] Sonnonstine T J and Perlman M M 1975 Surface-potential decay in insulators with fielddependent mobility and injection efficiency J. Appl. Phys. 46 3975-81

[4] Wintle H J 1972 Surface-charge decay in insulators with non-constant mobility and with deep trapping J.Appl.Phys. 43 2927-30

[5] Baum E A, Lewis T J and Toomer R 1977 Decay of electrical charge on polyethylene films $J$. Phys. D: Appl. Phys. 10 487-497

[6] Berlepsch H von 1985 Interpretation of surface potential kinetics in HDPE by a trapping model J. Phys. D: Appl. Phys. 18 1166-70

[7] Lutz B and Kindersberger J 2009 Determination of volume resistivity of polymeric insulators by surface charge decay Proceedings of the $16^{\text {th }}$ International Symposium on High Voltage Engineering, Innes House, Johannesburg. ISBN 978-0-620-44584-9

[8] Xu Z, Zhang L and Chen G 2007 Decay of electric charge on corona charged polyethylene $J$. Phys. D: Appl. Phys. 40 7085-9

[9] Lewiner J 1986 Evolution of experimental techniques for the study of the electrical properties of insulating materials IEEE Trans. Electr. Insul. 21 351-60

[10] Cherifi A, Abou-Dahka M and Toureille A 1992 The validation of the thermal step method IEEE Tran. Dielectr. Electr. Insul. 27 1152-8

[11] Li Y, Yasuda M and Takada T 1994 Pulsed electroacoustic method for measurement of charge accumulation in solid dielectrics IEEE Trans. Dielectr. Electr. Insul. 1 188-95

[12] Chen G, Xu Z and Zhang L 2007 Measurement of the surface potential decay of corona-charged polymer films using the pulsed electroacoustic method Meas. Sci. Technol. 18 1453-1458

[13] Roy S L, Segur P, Teyssedre G and Laurent C 2004 Description of bipolar charge transport in polyethylene using a fluid model with a constant mobility: model prediction J. Phys. D: Appl. Phys. 37 298-305

[14] Montanari G C, Mazzanti G, Palmieri F, Moton A, Perego G and Serra S 2001 Space-charge trapping and conduction in LDPE, HDPE and XLPE J. Phys. D: Appl. Phys. 34 2902-11

[15] Lide D R 2008 CRC handbook of Chemistry and Physics $89^{\text {th }}$ edition, p. 12-114

[16] Alison J M and Hill R M 1994 A model for bipolar charge transport trapping and recombination in degassed crosslinked polyethylene J. Phys. D: Appl. Phys. 27 1291-9

[17] Chen G 2010 A new model for surface potential decay of corona-charged polymers J. Phys. D: Appl. Phys. 43055405

[18] Chen G, Zhao J and Zhuang Y 2010 Numerical modelling of surface potential decay of corona charged polymeric material 2010 IEEE International Conference on Solid Dielectrics, Potsdam, Germany, 04 - 09 Jul 2010. IEEE, 549-552.

\section{Author biographies}




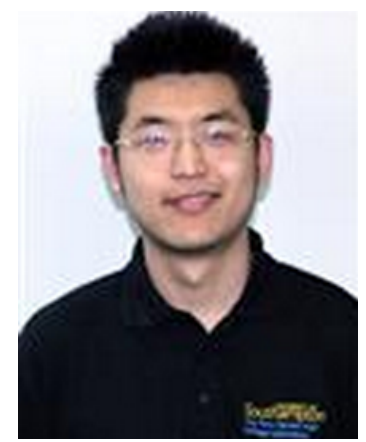

Miao Hao was born in China in 1987. He received his B.Eng. degree (2009) from Xi'an Jiaotong University, China. After he obtained his M.Sc. degree (2011) and Ph.D. degree (2015) from University of Southampton. He is now a postdoctoral research fellow in the University of Southampton. His main research interests include space charge and ageing mechanism in dielectrics for HVDC applications.

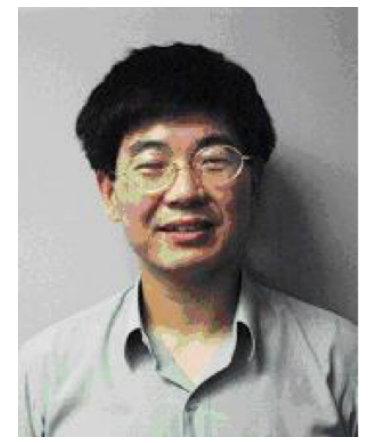

George Chen (SM'11) was born in China in 1961. He received his B.Eng (1983) and M.Sc (1986) degrees in electrical engineering from Xi'an Jiaotong University, China. After he obtained the Ph.D. degree (1990) from the University of Strathclyde, UK, on the permanent changes in the electrical properties of irradiated low-density polyethylene, he joined the University of Southampton as a postdoctoral research fellow and subsequently became a senior research fellow. In 1997 he was appointed as a research lecturer and promoted to a Reader in 2002 . He is now a professor of high voltage engineering at the University of Southampton and a visiting professor of Xi' an Jiaotong University. He has developed a wide range of interests in high voltage engineering and electrical properties of materials and published over 300 papers. He is active in HVDC systems and involved with technical working groups in both IEEE and CIGRE.

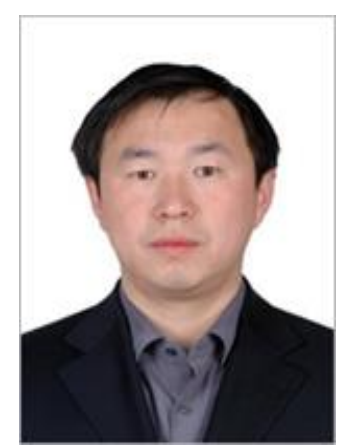

Xin Chen was born in 1973, He received the M.Sc. degree in 1998 and Ph.D. degree in 2001, from Southeast University. He now is a Professor research engineer of global energy interconnection research.

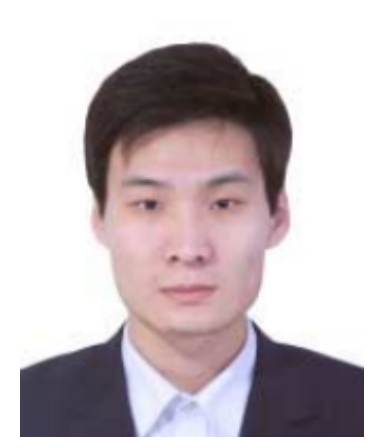

Chong Zhang was born in 1982. He received the M. Sc. Degree in physical chemistry of high polymers in 2007, from Shanghai Jiaotong University. He now is a senior research engineer of global energy interconnection research institute SGCC. His reseach interests are the dielecrtic insulation materials of transform and transmission. 


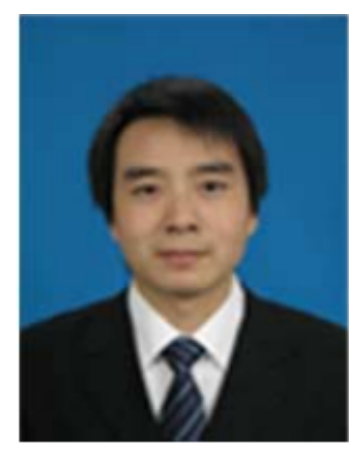

Wenpeng Li was born in Anhui Province in 1984. He received the M.Sc. degree in materials science and engineering in 2010, from Xi'an Jiaotong University. He now is a senior research engineer of global energy interconnection research institute SGCC. His research interests are the dielectric insulation materials of the HVDC cable.

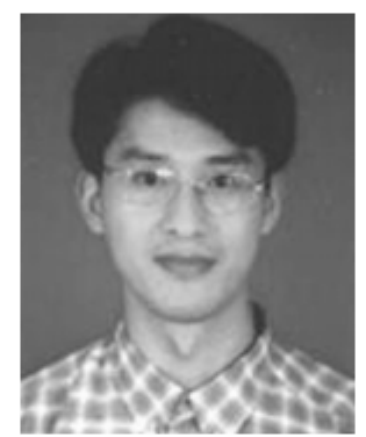

Haitian Wang (M'14) was born in Hunan, China. He received the B.Sc. degree in computation mathematics and the M. Eng. degree in electrical engineering from Sichuan University, Chengdu, China, in 1999 and 2003, respectively, and the Ph.D. degree in electrical engineering from Shanghai Jiaotong University, Shanghai, China in 2011. During 20112013, he had postdoctoral position with China Eletric Power Research Institute(CEPRI), Beijing, China. He was sponsored by the China Postdoctoral Science Foundation. In 2013, he joined the global energy interconnection research institute SGCC. His research fields include electromagnetic analysis, the VSC-HVDC transmission systems and HVDC cables. He is the author and coauthor of more than 20 scientific papers and, so far, has seven papers that have been published in IEEE TRANSACTIONS. He is a reveiewer for IEEE TRANSACTIONS ON POWER DELEVERY, INDUSTRY APPLICATIONS, and IEEE Industry Applications Magazine.

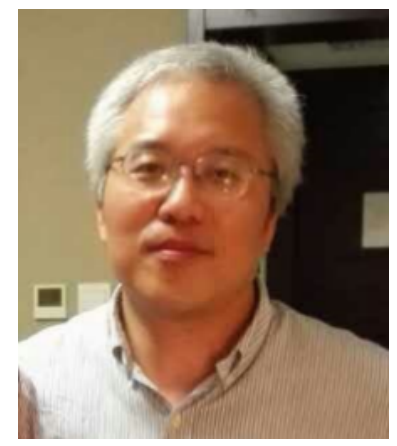

Mingyu Zhou received the Diplom-Ingenieur degree in electrical engineering from the University of Karlsruhe (now KIT), Germany and now is Ph.D. Candidate of Technische Universität Berlin (TUB), Germany. From 2009 to 2015 he worked with Südkabel (before 2004 with the name ABB Energiekabel $\mathrm{GmbH}$ ), germany and was responsible for development of cable accessories from $10 \mathrm{kV}-550 \mathrm{kV}$. Since October 2015 he is the Senior Engineer for HVDC Cablesystem with Global Energy Interconnection Reseach Institute Europe, Germany. His major research interest focus on development and diagnostics for high voltage cable system (AC\&DC).

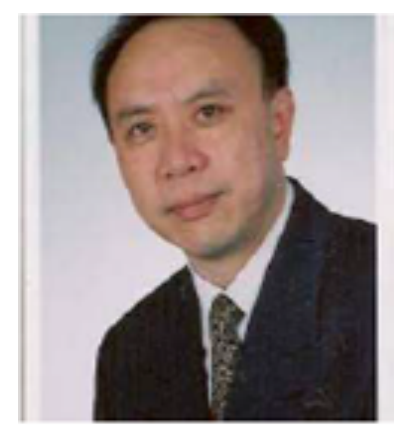

Xianzhang Lei, Director General, GEIRI Europe GmbH of State Grid Corporation of China. He received Ph.D from Technische Universität Berlin in Germany and continued his Post-Doctor at Yale University in USA. Afterwards he joined with Siemens AG in Germany and served as Vice President of Asia Pacific in Power System. Since 2010 he works with State Grid Corporation of China. Prof. Dr. Lei has 
published over 130 papers and possesses 14 patents in the fields of renewable energy, smart grid. He is a commissioner of "AC / DC coordination control professional group", high voltage $\mathrm{DC}$ and flexible $\mathrm{AC}$ transmission branch, CIGRE and commissioner of "power system planning and simulation professional group", high voltage power system branch, CIGRE. 